\title{
Treatment-related non-contiguous radiologic changes in children with diffuse intrinsic pontine glioma treated with expanded irradiation fields and antiangiogenic therapy
}

\author{
Zoltan Patay, MD, PhD. ${ }^{1,}$, Thomas E. Merchant, DO, PhD. ${ }^{2}$, Rosa Nguyen, MD. ${ }^{3}$, \\ Christopher R. Pierson, MD, PhD. ${ }^{4}$, Arzu Onar-Thomas, PhD. ${ }^{5}$, and Alberto Broniscer, MD. ${ }^{6}$ \\ ${ }^{1}$ Department of Diagnostic Imaging, St. Jude Children's Research Hospital, Memphis, TN \\ 2Department of Radiation Oncology, St. Jude Children's Research Hospital, Memphis, TN \\ ${ }^{3}$ Department of Oncology, St. Jude Children's Research Hospital, Memphis, TN \\ ${ }^{4}$ Department of Pathology and Laboratory Medicine, Nationwide Children's Hospital, Columbus, \\ $\mathrm{OH}$ \\ ${ }^{5}$ Department of Biostatistics, St. Jude Children's Research Hospital, Memphis, TN \\ ${ }^{6}$ Department of Pediatrics, University of Tennessee Health Science Center, Memphis, TN; \\ Department of Oncology, St. Jude Children's Research Hospital, Memphis, TN
}

\section{Introduction}

Children with diffuse intrinsic pontine glioma (DIPG) have a uniformly dismal prognosis. Currently, standard radiation therapy (RT) administered at cumulative doses of approximately 54 Gy by using daily fractionation is the mainstay of treatment.[1-4] Most contemporary clinical trials for children with DIPG extend the targeted volume for irradiation by approximately 1 to $1.5 \mathrm{~cm}$ beyond the boundaries of the T2/FLAIRhyperintense lesion area in all directions (clinical target volume, CTV) to account for subclinical microscopic infiltrative disease and add an additional margin of $0.3-0.5 \mathrm{~cm}$ to account for setup uncertainty.[2-4] Although contiguous infiltration of surrounding sites and leptomeningeal spread of DIPGs are known to occur, particularly later in the course of the disease,[5-8] most treatment failures are local in previously affected areas.[2, 9]

In an unpublished retrospective review of 37 patients with DIPG treated using 1-cm CTV and $0.3-0.5-\mathrm{cm}$ PTV margins, we found that $97 \%$ of all initial failures occurred within $2 \mathrm{~cm}$ of the caudal and transverse margins of the tumor and within $3 \mathrm{~cm}$ of its superior borders. On the basis of these data and following the completion of a phase I trial using a potent vascular endothelial growth factor receptor (VEGFR)-2 and modest epidermal growth factor receptor inhibitor (vandetanib) concurrently with RT using conventional 1-cm CTV and

\footnotetext{
*Corresponding author: Zoltan Patay, MD, St. Jude Children's Research Hospital, 262 Danny Thomas Place, Mail Stop 260, Memphis, TN, 38105, Telephone: 1-901-595-2507, fax: 1-901-595-3962, zoltan.patay@ stjude.org.

Conflict of interest: Dr. Alberto Broniscer received partial financial support from AstraZeneca to conduct the two clinical trials reported in this manuscript.
} 
0.3-0.5-cm PTV margins (Trial 1), we designed a clinical trial (Trial 2) to treat children with DIPG by combining conventionally fractionated local RT with expanded CTV margins (3$\mathrm{cm}$ cranial, 2-cm lateral and caudal) and two antiangiogenic agents: vandetanib and dasatinib administered concurrently.[11] Three patients enrolled on this clinical trial experienced obvious radiation necrosis at distant non-contiguous sites from the tumor at 5.5, 7, and 19.5 months after initiation of RT.[11] Although the development of necrotic changes within the tumor is a well-recognized phenomenon in children with DIPG, [12] the finding of non-contiguous treatment-related abnormalities (NCTRAs) was unusual.[11] We, therefore, retrospectively reviewed the clinical characteristics and serial brain MRIs of patients prospectively enrolled on two consecutive institutional Phase I clinical trials, Trial 1 (control cohort) and Trial 2 (study cohort).[4, 11] The purpose was to determine the occurrence of NCTRAs in each cohort and to investigate the putative mechanism of NCTRAs if significant differences between the two groups were found.

\section{Methods and Materials}

Trials 1 and 2 were consecutive institutional clinical trials combining concurrently administered local RT and antiangiogenic agents for children with DIPG. Trial 1 used standard CTV margins, and Trial 2 used expanded CTV margins.[4, 11] Both trials were approved by our Institutional Review Board, and consent for participation was obtained from patients' parents or legal guardians. For the purposes of this review, we requested that the board allow us to retrospectively evaluate some of the clinical, medication, and imaging data collected within the framework of the aforementioned clinical trials. This request, in conjunction with a waiver of repeated consenting, was granted.

Overall, 35 patients were enrolled in Trial 1, and 25 patients, in Trial 2. Five patients ( 3 from Trial 1 and 2 from Trial 2) were excluded from the current analysis because of a need for whole-brain RT due to tumor extension $(n=2)$, lack of volumetric RT planning data $(n=2)$, or a diagnosis other than DIPG (medullary glioblastoma, $n=1$ ). $[4,11]$ We, therefore, analyzed data from 23 patients (11 [48\%] girls; median age at diagnosis of 5.8 years; range: 2.3 to 17.2 years) treated on Trial 2, and 32 patients (18 [56\%] girls; median age at diagnosis of 6.4 years; range: 2.8 to 16.4 years) treated on Trial 1 . Only 1 patient remains alive 9.7 years after diagnosis. The 1-year progression-free and overall survival rates for Trials 1 and 2 were, respectively, $12.5 \pm 5.9 \%$ and $31.2 \pm 8.2 \%$ versus $30.4 \pm 9.6 \%$ and $52.2 \pm 10.4 \%$. The progression-free and overall survival rates of the trials were not statistically different (logrank $P=0.383$ and $P=0.744$, respectively).

Patients enrolled on Trial 2 received escalating doses of vandetanib and dasatinib concurrently with and following the completion of RT.[11] Local RT was administered at a dose of 54 Gy in 1.8-Gy fractions. The CTV comprised the gross tumor volume (GTV) as determined by the extent of hyperintense signal abnormalities in T2-weighted and/or FLAIR images, with additional respective extensions of $2 \mathrm{~cm}, 2 \mathrm{~cm}$, and $3 \mathrm{~cm}$ in the transverse, caudal, and cranial directions.[11] The planning target volume (PTV) contained a $0.3-\mathrm{cm}$ extension in all directions to account for variations in position.[11] 
Patients enrolled on Trial 1 were treated with escalating doses of vandetanib concurrently with and following RT.[4] RT in this clinical trial was similar to that in Trial 2 except for the use of a smaller CTV with 1-cm margins in all directions and variable 0.3-0.5-cm PTV margins.[4] The reduction in the PTV margin resulted from the use of cone-beam CT image guidance in Trial 2. RT volumes and dosimetry were assessed by using commercially available software (MIM Software Inc., Cleveland, $\mathrm{OH}$ ).

In both studies, brain MRI examinations were obtained before the start of therapy, at completion of RT, and every 8 to 10 weeks thereafter for the duration of treatment. $[4,11]$ Follow-up examinations were performed approximately every 3 months after completion of therapy. All MRI studies were performed on 3T magnets and always included pre-contrast sagittal and axial T1-weighted sequences, pre-contrast axial T2-weighted sequences, postcontrast sagittal and axial T1-weighted FLAIR sequences, and post-contrast axial T2weighted FLAIR sequences. A few follow-up studies (typically late) were performed in other institutions. Imaging protocols in such instances were inconsistent and differed from ours, but we used those studies when it was useful for our purposes.

A review of brain MRIs of all patients was conducted by a neuroradiologist who did not see the clinical data. The RT planning of all patients was reviewed by a radiation oncologist and neuro-oncologist to verify compliance with treatment guidelines and to correlate RT isodose with the location of NCTRAs. Multi-planar multi-sequence imaging series were registered to the radiation therapy treatment planning $\mathrm{CT}$ and data. NCTRAs were evaluated with respect to the isodose volumes.

Statistical analysis was performed by using single-variable approaches, evaluating the association between potential covariates and NCTRAs in patients enrolled on both clinical trials. Standard descriptive approaches were used for cohort characterization. The KruskalWallis test or Fisher's exact test were used as appropriate to evaluate associations of covariates with protocol and presence or absence of NCTRAs. Survival comparisons were based on log-rank tests. A $P$ value of $\leq 0.05$ was regarded as being statistically significant.

\section{Results}

MR imaging findings were classified according to a 5-category scheme (Types 0-IV)

Type 0: Abnormalities confined to the primary lesion field in the pons, occasionally extending to immediate adjacent areas (midbrain, middle cerebellar peduncles, etc.) but remaining contiguous with the primary tumor.

Type I: New (i.e., not present on earlier studies), often symmetrical T2-hyperintense lesions involving cerebellum (typically upper vermis, cerebellar tonsils, anterior aspects of cerebellar hemispheres). These lesions usually did not show signal enhancement on post-contrast T1-weighted images and were associated with swelling of the involved structures. (Fig.1 and 2)

Type II: Supratentorial lesions involving deep gray matter structures (thalami, globi pallidi, putamina, caudate nuclei), and/or white matter structures (typically subinsular 
regions, mesial temporal structures, inferomedial temporal-occipital regions). (Fig. 1 and 2)

Type III: Supratentorial, extensive "leukoencephalopathy-type" changes within periand supraventricular white matter. (Fig. 3)

Type IV: Supratentorial infarction (deep watershed territory or cortical). (Fig. 4)

Types I-IV represent the different (non-sequential) imaging phenotypes of NCTRAs observed in our patients. Type I, II, and III lesions were found to present in isolation or in combination and were not always synchronous in their appearance. When Type I (infratentorial) and Type II (supratentorial) lesions developed, Type II lesions were seen first followed by Type I lesions several months later in 2 patients. In another patient, Type I and II lesions developed simultaneously. In 1 patient, Type I lesions were biphasic. (Fig. 2) NCTRAs on brain MRIs usually occurred at a median of 7 months (range: 0.7 to 19.5 months) after the start of therapy (Table 1). They developed in 9 of 23 (39\%) of the Trial 2 patients and in none of the Trial 1 patients. Four of these 9 patients (44\%) presented with new clinical signs and symptoms associated with the first imaging appearance of the NCTRAs.

NCTRAs were classified according to their location, extent, and other imaging characteristics (Table 1). Three patients (patients 1,2, and 5) had concomitant or sequential infra- and supratentorial NCTRAs. (Fig. 1 and 2) Three patients (patients 1, 2, and 4) had new supratentorial $\mathrm{T} 2$-weighted hyperintense lesions, with foci of signal enhancement in postcontrast T1-weighted images in areas uninvolved by the tumor, suggestive of radiation necrosis (Table 1).[11] Patients 1 and 2 experienced clinical and radiologic improvement of radiation necrosis before tumor progression; radiation necrosis was detected simultaneously with tumor progression in patient 4 . Two patients (patients 4 and 9) had supratentorial NCTRAs involving the deep gray matter (thalami, globi pallidi, putamina, caudate nuclei) and/or white matter (subinsular, mesial temporal, inferomedial temporo-occipital regions). Isolated T2-hyperintense non-enhancing lesions in the cerebellum developed in 1 patient (patient 3). Extensive supratentorial peri- and supraventricular white matter changes reminiscent of leukoencephalopathy (Fig. 3) occurred in 2 patients (patients 6 and 8). Asymptomatic subtle lacunar infarcts in the left frontal lobe developed in 1 patient (patient 7) 12.5 months after initiation of therapy and concurrently with local tumor progression. (Fig. 4) This patient had a left frontal lobe ischemic stroke 3 weeks later while receiving palliative therapy with oral etoposide; she underwent a comprehensive thrombophilic evaluation that showed heterozygosity for factor V Leiden mutation. After her death due to tumor progression 8 months later, an autopsy revealed cerebral arterial vasculopathy that focally occluded the lumen of the anterior cerebral and left vertebral arteries and reduced the lumen of both middle and posterior cerebral arteries and the right vertebral artery. (Fig. 5).

Children with NCTRAs were younger (median age at diagnosis of DIPG, 3.7 vs. 6.4 years; Kruskal-Wallis $P=0.003$ ). These patients had larger volumes of brain exposed to higher radiation doses, as determined by comparing the whole-brain isodose volumes $\geq 20 \mathrm{~Gy}$. Patients in whom NCTRAs developed also had larger relative CTV $(P=0.009)$ and PTV $(P=0.025)$ values, as determined by the ratio of CTV or PTV to whole-brain volume. Within 
the Trial 2 cohort, the relative GTV normalized to whole-brain volume was significantly larger in patients with NCTRAs than in those without such changes $(P=0.014)$. Patients with NCTRAs treated on Trial 2 received doses of dasatinib and vandetanib similar to those of non-affected patients (Fisher exact $P=0.116$ and 0.537 , respectively). They also remained on treatment for a longer period of time (385 vs. 210 days; Kruskal-Wallis $P=0.044$ ).

In all patients except 1 (patient 6), NCTRAs developed within the CTV. However, among patients treated on Trial 2, RT planning of 9 patients (39\%) was found to be a posteriori not compliant with protocol requirements. Specifically, CTV was set at a smaller margin, most commonly at $1 \mathrm{~cm}$ from the GTV, particularly in the cranial direction. Yet, NCTRAs (within the inadvertently reduced CTV) developed within 6 of these patients. Although the territory of infarction in patient 7 was within the 20-Gy isodose, the steno-occlusive changes involving the left proximal anterior and middle cerebral arteries found at autopsy were within the CTV (Figure 4). Treatment plans were reviewed to assess the dose to the major or central components of arterial circulation including the basilar and carotids arteries and Circle of Willis. With the entire pons forming the basis of the gross-tumor volume and the clinical target volume anatomically-constrained at the intracranial margin of the base of skull, the dose to the basilar artery and posterior aspect of the Circle of Willis was found to be uniformly exposed to the prescription dose (54Gy). The dose to the carotid arteries and more anterior components of the Circle of Willis were uniformly exposed to doses in the range of 50-54Gy. The lack of variability in dose to these structures reduces their value as organs at risk (OAR). Patient 6 was noted to have extensive supratentorial leukoencephalopathy, which was contained within areas cumulatively exposed to approximately $28 \mathrm{~Gy}$.

\section{Discussion}

Although new imaging changes (necrosis, hemorrhage, contrast uptake) within the tumor lesion commonly develop in children with DIPG after RT with or without concomitant treatments, $[12,13]$ to our knowledge, this is the first report to describe a high incidence of changes in distant non-contiguous brain areas without prior imaging evidence of tumor involvement. Our review of brain MRI data and RT plans suggests that the genesis of NCTRAs in our patient cohort was multifactorial. RT seemed to have played a major role in the occurrence of NCTRAs because their development was associated with brain tissue exposure to at least $28 \mathrm{~Gy}$, but predominantly to $54 \mathrm{~Gy}$ (CTV). Despite the protocol stipulations for RT with expanded volumes in patients enrolled in Trial 2, we found that 39\% of those patients, including two-thirds of those in whom NCTRAs developed, had a noncompliant, limited CTV expansion, most commonly corresponding to only a 1-cm margin cranially, which is similar to that used in Trial 1 and in most other contemporary clinical trials for children with DIPG. The potential side effects of RT in the central nervous system, including small- or large-vessel angiopathy and resultant or independent leukoencephalopathy have long been recognized.[14] However, because similar radiologic abnormalities have not been reported in other clinical trials for children with DIPG, including those conducted before the widespread availability of conformal techniques, we hypothesize that NCTRAs in our patients cannot be attributed solely to RT. 
Current clinical trials for children with DIPG commonly combine RT with small-molecule inhibitors.[15] Both clinical trials described in this manuscript used vandetanib, a potent VEGFR-2 inhibitor.[4, 11] On the basis of the design of the studies, patients on Trial 1 received, on average, much higher doses of vandetanib than did those treated on Trial 2.[4, 11] Pharmacodynamic studies performed in peripheral-blood mononuclear cells in patients treated on Trial 1 showed a more robust inhibition of the VEGF pathway in those treated at the highest dosage level of vandetanib, which was double the dose received by most patients in Trial 2. [4] VEGF is associated with increased vascular permeability, endothelial cell proliferation, and angiogenesis.[16-18] Bevacizumab, a humanized monoclonal antibody targeting VEGF has been successfully used in the treatment of radiation necrosis in children and adults. $[19,20]$ Based on the lack of NCTRAs in patients treated in trial 1 and the use of VEGF inhibitors to treat radiation necrosis, it is counterintuitive to believe that vandetanib had any direct role in the genesis of these treatment-related changes.

Dasatinib is a multi-tyrosine kinase inhibitor with potent activity against PDGFRA and B, Src, and c-Kit.[21, 22] The PDGF pathway is crucial in regulating the function of pericytes, which play key roles by interacting with endothelial cells, contributing to the stability of capillary walls, regulating the cerebral blood flow, and maintaining an intact blood-brain barrier.[23, 24] The PDGF pathway has also been implicated in accelerated endothelial and smooth muscle cell proliferation in preclinical models of capillary injury.[25, 26] Although dasatinib and other PDGFR inhibitors have shown an increased anticancer effect in preclinical cancer models when combined with RT, including in models of glioblastoma, [27, 28]there are no pre-clinical data to suggest a potential for increased toxicity of this combination to normal brain tissue and endothelium. To our knowledge, only 1 small clinical trial showed a potential for increased toxicity when dasatinib was administered concurrently with RT. [29] In that study, 2 of 11 patients with lung cancer treated with local $\mathrm{RT}$ and dasatinib at a maximum dose of $70 \mathrm{mg}$ per day experienced either grade 3 or 5 pneumonitis. Furthermore, we have conducted a second clinical trial combining RT and crenolanib, a potent and specific PDGFR inhibitor, in children with DIPG and have not observed similar radiologic abnormalities. (unpublished personal data)] Overall, we can only speculate that dasatinib inhibition of PDGFR and/or some of its other targets in combination with RT may have contributed to the formation of NCTRAs.

Histologic evaluation of the NCTRAs at autopsy was possible in only 1 of our patients, who experienced a stroke. RT can cause injury to small, mid-sized, and large vessels and is a known cause of premature atherosclerosis.[14, 30] However, the widespread arterial abnormalities found in our patient and the short latency from RT were remarkable. Although there is no consensus in the literature,[31] several studies support an association between factor V Leiden heterozygosity and increased risk for strokes.[32-34] We believe that the presence of factor $\mathrm{V}$ Leiden heterozygosity in addition to treatment-related risk factors led to the development of early, severe, large-vessel arteriopathy and stroke in this patient.

In addition to the patient who experienced an ischemic stroke, the pattern and location of most other NCTRAs (watershed areas and areas supplied by terminal/perforating arteries) lead us to suspect that impaired microcirculation in vascular territories without readily 
available collateral blood flow pathways may be the "common denominator" in our cases and be the principal mechanism responsible for these complications.

Younger children are more vulnerable to the toxicities of RT.[35] The association of NCTRAs and younger age in our Trial 2 cohort may represent additional proof of this increased vulnerability. Younger children with DIPG have a somewhat better prognosis (i.e. longer overall survival).[36, 37] Indeed, despite the treatment-related comorbidity burden, the median survival of patients with NCTRAs was 1.7 years, significantly longer than that expected for children with DIPG. However, it is also possible that some of the NCTRAs were detected simply because those patients lived longer.

Several recent studies have advocated the potential benefits of expanding the RT fields in children with DIPG because of the characteristic treatment-failure patterns along the primary lesion margins and the high incidence of contiguous infiltrative and leptomeningeal tumor spread.[7, 8]. To our knowledge, our Trial 2 was the first to use multiple non-conventional agents concurrent with irradiation in the treatment of DIPG and in the setting of expanded CTV compared to conventional RT. When RT (with or without expanded CTV) is used in combination with agents (especially more than one), including small-molecules with antiangiogenic effects, the risk of unusual neurotoxicity as a result of the poorly understood, possibly complex interplay between the individually tolerable but synergistically acting agents should be considered; hence, such patients require close clinical and imaging monitoring, limited target volume margins, and increased attention paid to target dose heterogeneity.

Despite some differences in therapy between both studies, NCTRAs occurred predominantly in young patients with large tumors who were treated with expanded RT volumes in combination with potent antiangiogenic therapy.[11] On the basis of the characteristics and location of the lesions, we speculate that vascular insult secondary to the combination of RT with more-potent antiangiogenic therapy in the vulnerable population of younger children with larger tumors may have played a role in the genesis of NCTRAs. Therefore, we cannot attribute the occurrence of these treatment-related changes only to the use of expanded RT fields because two-thirds of patients experiencing this phenomenon were treated with conventional treatment expansions. The lack of NCTRAs in Trial 1 suggests that an interaction between dasatinib and RT played an important role in the development of NCTRAs.

\section{Supplementary Material}

Refer to Web version on PubMed Central for supplementary material.

\section{Acknowledgments}

The authors thank Dr. Cherise Guess of the Department of Scientific Editing at St. Jude Children's Research Hospital for editing the manuscript and Edwina Anderson for data management.

Funding: This work was supported by the U.S. National Institutes of Health Cancer Center Support (CORE) Grant P30 CA21765, by the American Lebanese Syrian Associated Charities (ALSAC), and by a grant from AstraZeneca. 


\section{References}

1. Hargrave D, Bartels U, Bouffet E. Diffuse brainstem glioma in children: critical review of clinical trials. Lancet Oncol. 2006; 7(3):241-8. [PubMed: 16510333]

2. Mandell LR, et al. There is no role for hyperfractionated radiotherapy in the management of children with newly diagnosed diffuse intrinsic brainstem tumors: results of a Pediatric Oncology Group phase III trial comparing conventional vs. hyperfractionated radiotherapy. Int J Radiat Oncol Biol Phys. 1999; 43(5):959-64. [PubMed: 10192340]

3. Bradley KA, et al. Motexafin-gadolinium and involved field radiation therapy for intrinsic pontine glioma of childhood: a children's oncology group phase 2 study. International Journal of Radiation Oncology, Biology, Physics. 2013; 85(1):e55-60.

4. Broniscer A, et al. Phase I study of vandetanib during and after radiotherapy in children with diffuse intrinsic pontine glioma. J Clin Oncol. 2010; 28(31):4762-8. [PubMed: 20921456]

5. Donahue B, et al. Patterns of recurrence in brain stem gliomas: evidence for craniospinal dissemination. Int J Radiat Oncol Biol Phys. 1998; 40(3):677-80. [PubMed: 9486619]

6. Gururangan S, et al. Incidence and patterns of neuraxis metastases in children with diffuse pontine glioma. J Neurooncol. 2006; 77(2):207-12. [PubMed: 16568209]

7. Caretti V, et al. Subventricular spread of diffuse intrinsic pontine glioma. Acta Neuropathol. 2014; 128(4):605-7. [PubMed: 24929912]

8. Buczkowicz P, et al. Histopathological spectrum of paediatric diffuse intrinsic pontine glioma: diagnostic and therapeutic implications. Acta Neuropathol. 2014; 128(4):573-81. [PubMed: 25047029]

9. Grigsby PW, Garcia DM, Ghiselli R. Analysis of autopsy findings in patients treated with irradiation for thalamic and brain stem tumors. Am J Clin Oncol. 1989; 12(3):255-8. [PubMed: 2543206]

10. Chang A, Merchant T. Patterns of failure for diffuse infiltrating brainstem glioma: new guidelines for radiotherapy [abstract]. Neuro Oncol. 2008; 10(3):1. [PubMed: 18184773]

11. Broniscer A, et al. Phase I trial, pharmacokinetics, and pharmacodynamics of vandetanib and dasatinib in children with newly diagnosed diffuse intrinsic pontine glioma. Clin Cancer Res. 2013; 19(11):3050-8. [PubMed: 23536435]

12. Packer RJ, et al. Early cystic/necrotic changes after hyperfractionated radiation therapy in children with brain stem gliomas. Data from the Childrens Cancer Group. Cancer. 1993; 71(8):2666-74. [PubMed: 8453590]

13. Hargrave D, Chuang N, Bouffet E. Conventional MRI cannot predict survival in childhood diffuse intrinsic pontine glioma. J Neurooncol. 2008; 86(3):313-9. [PubMed: 17909941]

14. Perry A, Schmidt RE. Cancer therapy-associated CNS neuropathology: an update and review of the literature. Acta Neuropathol. 2006; 111(3):197-212. [PubMed: 16463065]

15. Kaye EC, Baker JN, Broniscer A. Management of diffuse intrinsic pontine glioma in children: current and future strategies for improving prognosis. CNS Oncol. 2014; 3(6):421-31. [PubMed: 25438813]

16. Leung DW, et al. Vascular endothelial growth factor is a secreted angiogenic mitogen. Science. 1989; 246(4935):1306-9. [PubMed: 2479986]

17. Keck PJ, et al. Vascular permeability factor, an endothelial cell mitogen related to PDGF. Science. 1989; 246(4935):1309-12. [PubMed: 2479987]

18. Jain RK. Antiangiogenesis strategies revisited: from starving tumors to alleviating hypoxia. Cancer Cell. 2014; 26(5):605-22. [PubMed: 25517747]

19. Levin VA, et al. Randomized double-blind placebo-controlled trial of bevacizumab therapy for radiation necrosis of the central nervous system. Int J Radiat Oncol Biol Phys. 2011; 79(5):148795. [PubMed: 20399573]

20. Liu AK, Macy ME, Foreman NK. Bevacizumab as therapy for radiation necrosis in four children with pontine gliomas. International Journal of Radiation Oncology, Biology, Physics. 2009; 75(4): 1148-54. 
21. Chen Z, et al. Potent inhibition of platelet-derived growth factor-induced responses in vascular smooth muscle cells by BMS-354825 (dasatinib). Mol Pharmacol. 2006; 69(5):1527-33. [PubMed: 16436588]

22. Lombardo LJ, et al. Discovery of N-(2-chloro-6-methyl- phenyl)-2-(6-(4-(2-hydroxyethyl)piperazin-1-yl)-2-methylpyrimidin-4- ylamino)thiazole-5-carboxamide (BMS-354825), a dual $\mathrm{Src} / \mathrm{Abl}$ kinase inhibitor with potent antitumor activity in preclinical assays. Journal of Medicinal Chemistry. 2004; 47(27):6658-61. [PubMed: 15615512]

23. Benjamin LE, Hemo I, Keshet E. A plasticity window for blood vessel remodelling is defined by pericyte coverage of the preformed endothelial network and is regulated by PDGF-B and VEGF. Development. 1998; 125(9):1591-8. [PubMed: 9521897]

24. Trost A, et al. Brain and Retinal Pericytes: Origin, Function and Role. Front Cell Neurosci. 2016; 10:20. [PubMed: 26869887]

25. Myit S, et al. Different growth properties of neointimal and medial smooth muscle cells in response to growth factors. J Vasc Res. 2003; 40(2):97-104. [PubMed: 12808345]

26. Boor P, et al. PDGF-C mediates glomerular capillary repair. Am J Pathol. 2010; 177(1):58-69. [PubMed: 20489153]

27. Timke C, et al. Combination of vascular endothelial growth factor receptor/platelet-derived growth factor receptor inhibition markedly improves radiation tumor therapy. Clinical Cancer Research. 2008; 14(7):2210-9. [PubMed: 18381963]

28. Raju U, et al. Dasatinib, a multi-kinase inhibitor increased radiation sensitivity by interfering with nuclear localization of epidermal growth factor receptor and by blocking DNA repair pathways. Radiotherapy \& Oncology. 2012; 105(2):241-9. [PubMed: 23010482]

29. Khurshid H, et al. A Phase I Study of Dasatinib with Concurrent Chemoradiation for Stage III Non-Small Cell Lung Cancer. Front Oncol. 2012; 2:56. [PubMed: 22666662]

30. Stewart FA, Hoving S, Russell NS. Vascular damage as an underlying mechanism of cardiac and cerebral toxicity in irradiated cancer patients. Radiat Res. 2010; 174(6):865-9. [PubMed: 21128810]

31. Laugesaar R, et al. Factor V Leiden and prothrombin 20210G >A [corrected] mutation and paediatric ischaemic stroke: a case-control study and two meta-analyses. Acta Paediatr. 2010; 99(8):1168-74. [PubMed: 20337781]

32. Olcay L, et al. Cerebral infarct associated with factor V Leiden mutation in a boy with hemophilia A. Am J Hematol. 1997; 56(3):189-90. [PubMed: 9371534]

33. Kenet G, et al. Factor V Leiden and antiphospholipid antibodies are significant risk factors for ischemic stroke in children. Stroke. 2000; 31(6):1283-8. [PubMed: 10835445]

34. Rosenbloom AL. Fatal cerebral infarctions in diabetic ketoacidosis in a child with previously unknown heterozygosity for factor V Leiden deficiency. J Pediatr. 2004; 145(4):561-2. [PubMed: 15480386]

35. Fouladi M, et al. Intellectual and functional outcome of children 3 years old or younger who have CNS malignancies. J Clin Oncol. 2005; 23(28):7152-60. [PubMed: 16192599]

36. Broniscer A, et al. Young age may predict a better outcome for children with diffuse pontine glioma. Cancer. 2008; 113(3):566-72. [PubMed: 18484645]

37. Khuong-Quang DA, et al. K27M mutation in histone H3.3 defines clinically and biologically distinct subgroups of pediatric diffuse intrinsic pontine gliomas. Acta Neuropathol. 2012; 124(3): 439-47. [PubMed: 22661320] 

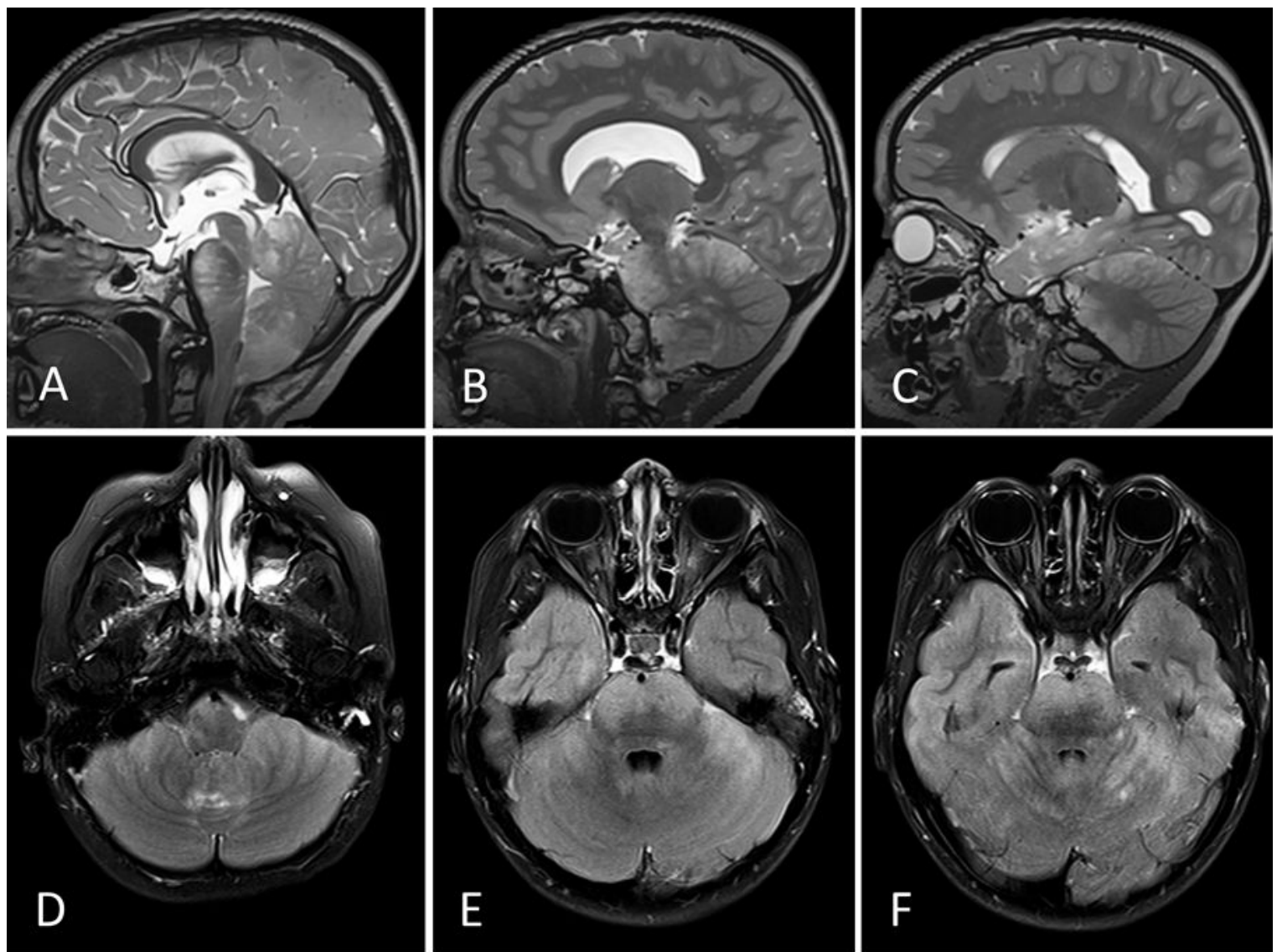

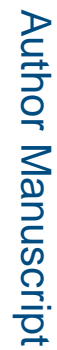
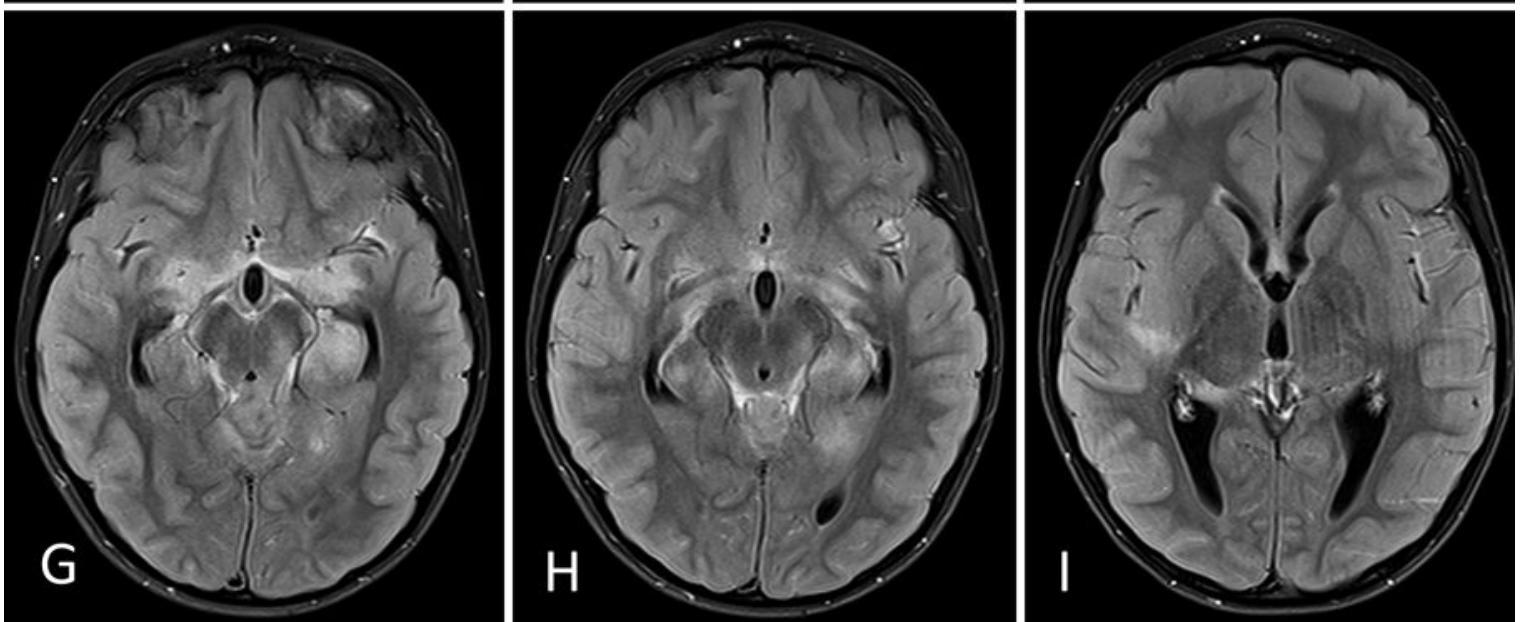

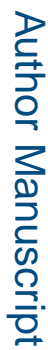

Fig. 1.

(Patient 5) MRI evaluation of a patient in whom cerebellar (type I) and supratentorial (type II) lesions developed outside the tumor field during the course of treatment. Sagittal T2weighted images (A-C) and axial contrast-enhanced FLAIR images of the infratentorial (DF) and supratentorial (G-I) brain that were acquired 13 months after diagnosis. T2hyperintensities are seen within the brainstem at the level of the pons, consistent with residual tumor (A-B, E-F). T2-hyperintensities are also shown within cerebellum at the level of the upper vermis and the cerebellar tonsils (A), cerebellar hemispheres anteriorly and 
superficially involving the cortex and immediate subcortical white matter and associated with swelling of the involved structures (B-F). Supratentorially, abnormalities are present within mesial temporal structures, in the anterior perforate areas (G-H), in the left inferomedial temporal-occipital junction area $(\mathrm{H})$ and the right posterior peri-insular region (I). These lesions were less prominent but conspicuous on the previous MRI study 2 months earlier. 

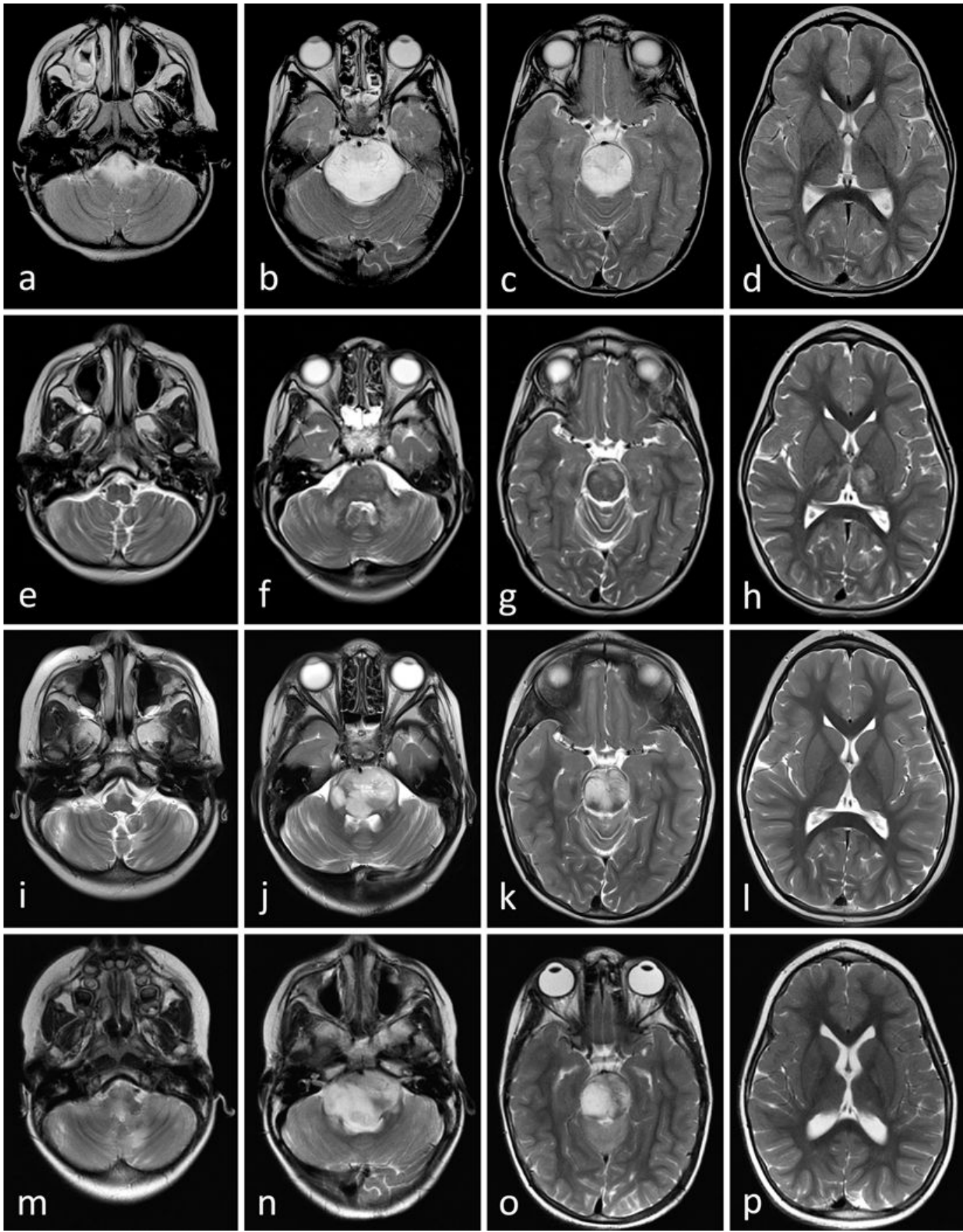

Fig. 2.

(Patient 2) MRI of Type II and biphasic Type I lesions. Transverse T2-weighted images acquired at the time of the initial diagnostic examination (A-D) and at 6-month (E-H), 12 month (I-L), and 14-month (M-P) follow-up examinations. The 6-month follow-up study shows abnormalities within the middle cerebellar peduncles and deep cerebellar white matter bilaterally (Type I lesions) as well as within thalami and posterior limbs of the internal capsules (Type II lesions). These lesions almost totally resolved subsequently; only subtle signal changes are suggested within left globus pallidus (L) on the 12-month follow- 
up study. The 14-month examination shows new Type I lesions in the posterior fossa, including the anterior-inferior cerebellar hemispheres $(\mathrm{M})$ and the upper vermis $(\mathrm{O})$ and presenting with diffuse swelling of the cerebellum (M-N). 

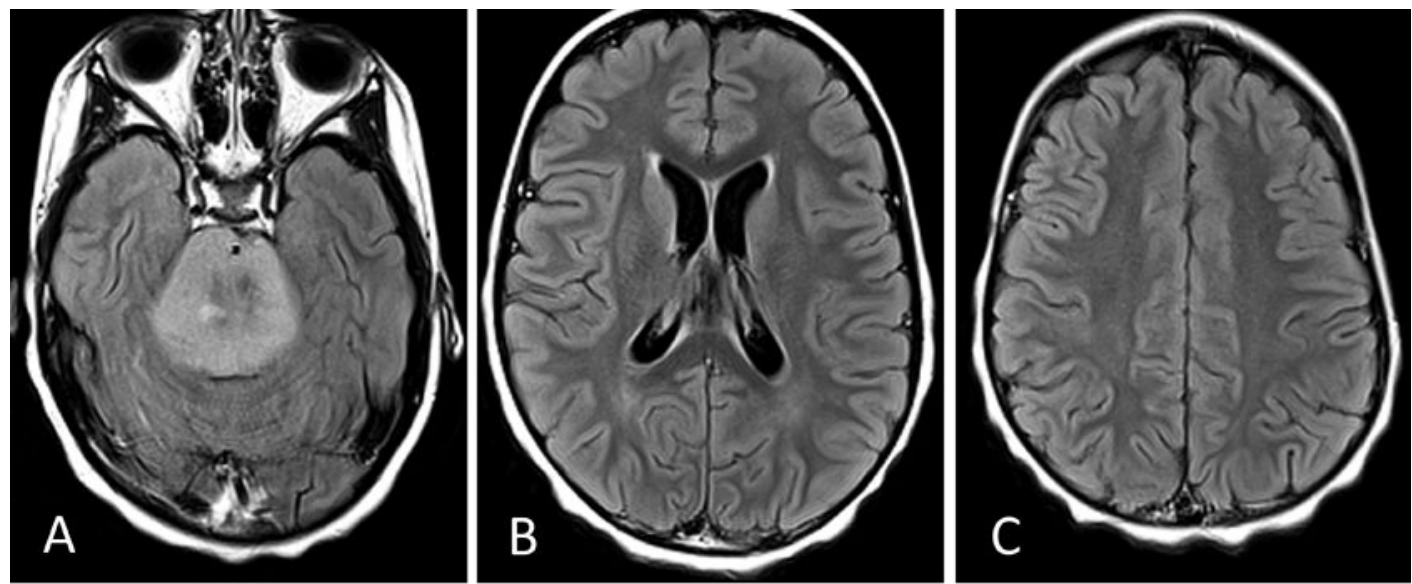

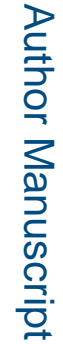
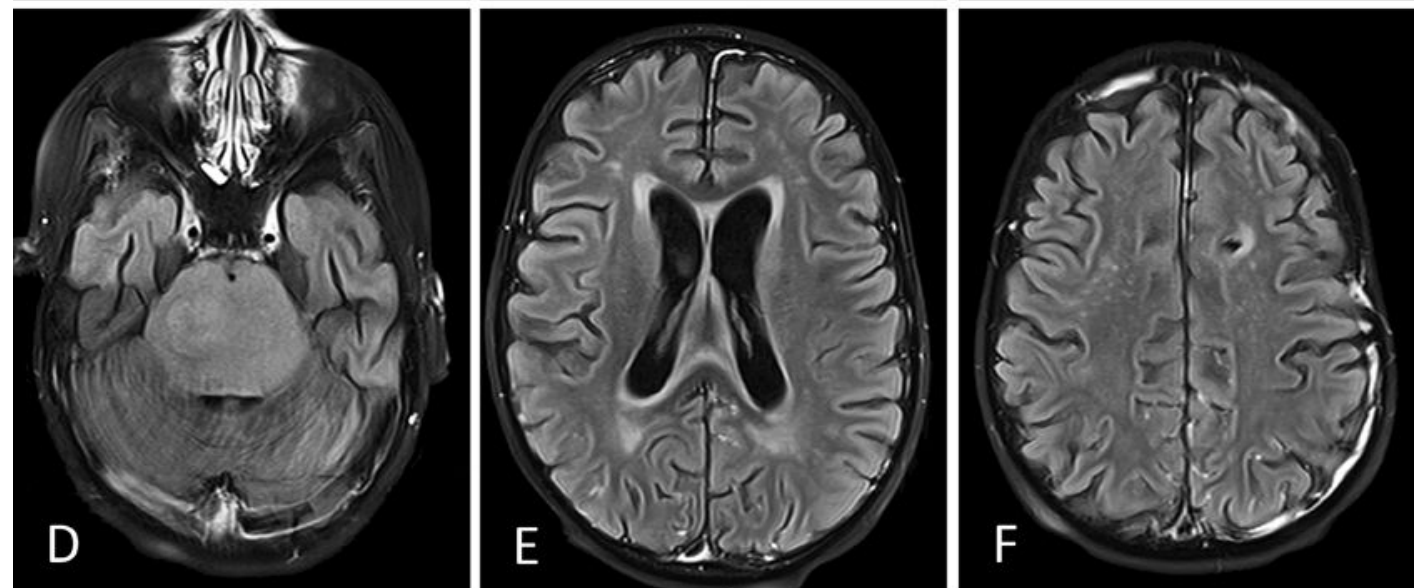

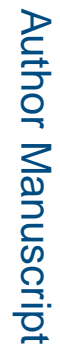
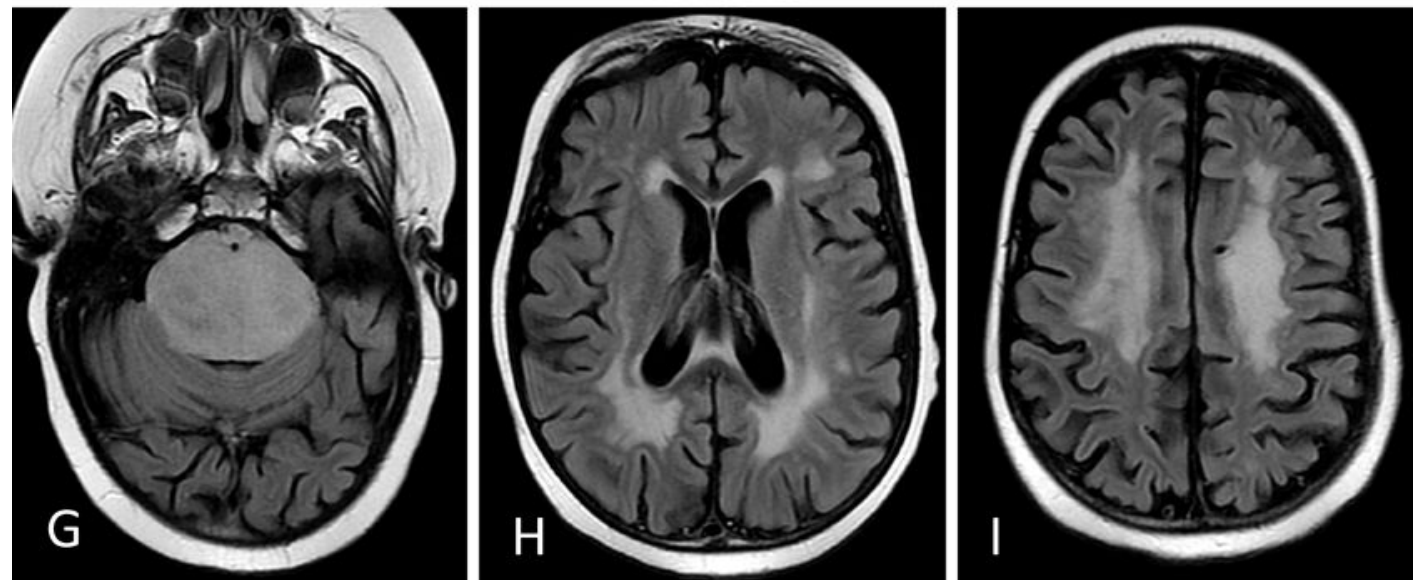

Fig. 3.

(Patient 6) Longitudinal MRI evaluation of a patient treated for DIPG in whom supratentorial leukoencephalopathy (Type III lesion) developed during treatment. Axial T2weighted FLAIR images at baseline (A-C), at 6-week (D-F) and at 7-month (G-I). Initially, no definite abnormalities are seen within supratentorial white matter (B-C). At 6 weeks, modest periventricular hyperintensities appear in the frontal and parietal regions $(\mathrm{E})$, and clusters of small punctate hyperintensities are also suggested in the frontal region. 


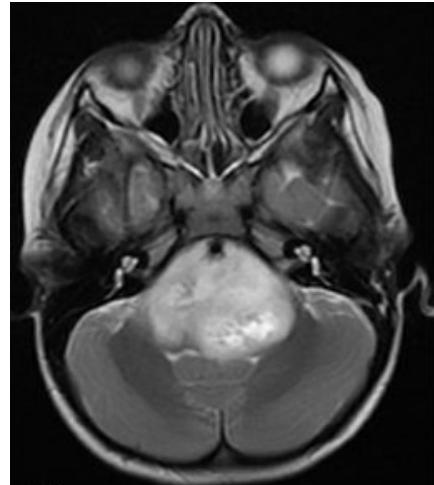

a

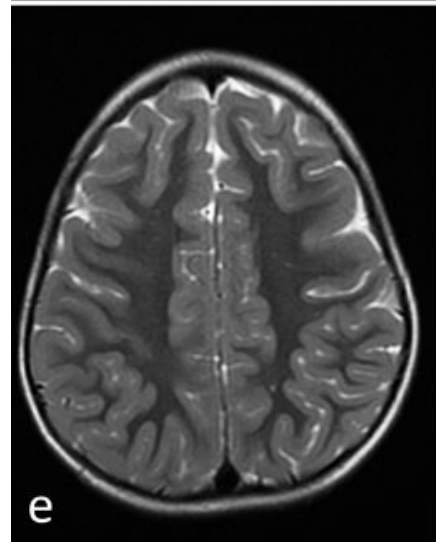

b
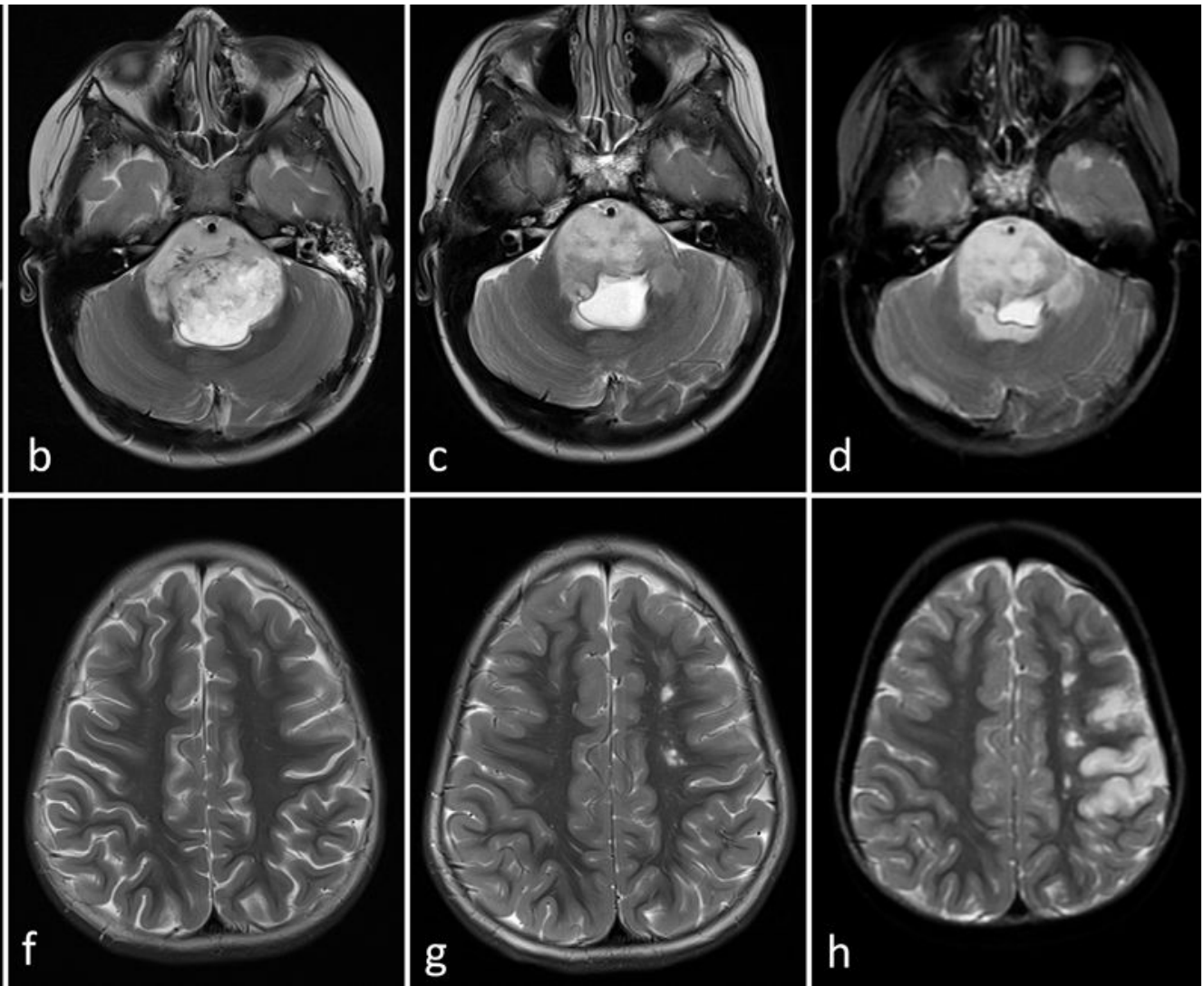

Fig. 4.

(Patient 7) MRI of Type IV lesion. Transverse T2-weighted images of the posterior fossa (AD) at initial diagnostic examination (A), 3 weeks later (B), at 13-month (C) and at 14-month (D) and of the supratentorial brain (E-H) at the same time points. The tumor initially progressed (B), then remained gross stable for the following year. At 13 months, deep watershed territory infarctions appeared in the left cerebral hemisphere $(\mathrm{G})$; and a month later, additional cortical infarction developed in the left middle cerebral artery territory $(\mathrm{H})$. 


\begin{tabular}{|c|c|c|c|c|c|c|c|c|c|}
\hline 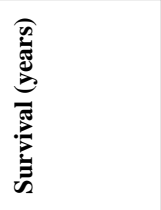 & 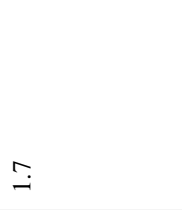 & $\stackrel{ \pm}{-}$ & 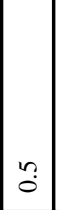 & 2 & $\stackrel{\infty}{-}$ & $\dot{o}$ & a & $\stackrel{\infty}{\circ}$ & $m$ \\
\hline 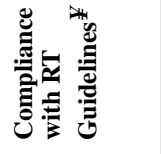 & 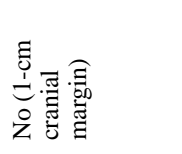 & 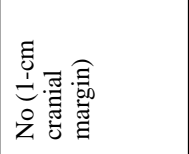 & $\approx$ & 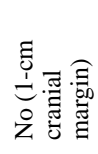 & $\check{z}$ & ฉ & 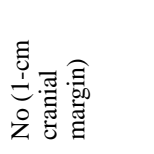 & 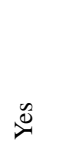 & 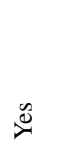 \\
\hline 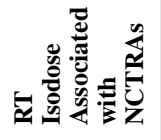 & 触 & 触 & $\mid \begin{array}{l}3 \\
0 \\
\vdots \\
i\end{array}$ & 苦 & 触 & $\begin{array}{l}\vec{j} \\
\infty \\
\dot{0} \\
\dot{N}\end{array}$ & $\begin{array}{l}\overrightarrow{0} \\
\text { 品 }\end{array}$ & 总 & 离 \\
\hline 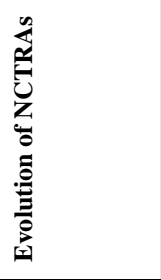 & 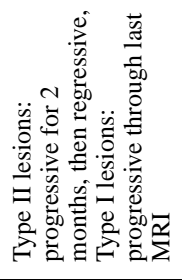 & 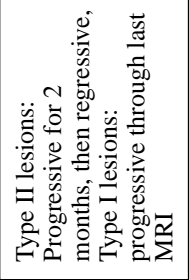 & 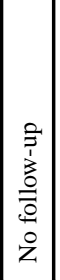 & 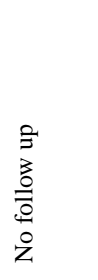 & 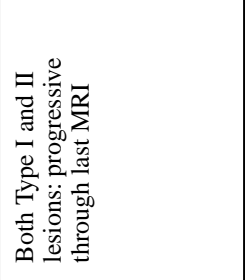 & 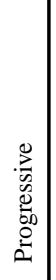 & $\begin{array}{l}0 \\
0 \\
0 \\
0 \\
0 \\
0 \\
0 \\
z\end{array}$ & 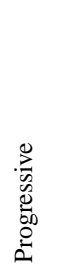 & 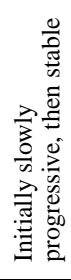 \\
\hline 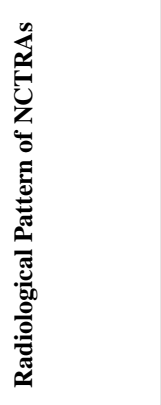 & 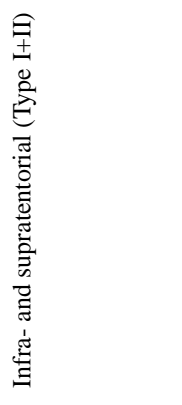 & 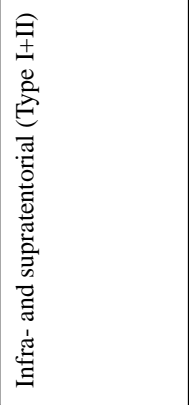 & 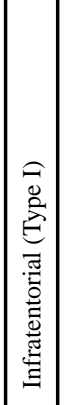 & 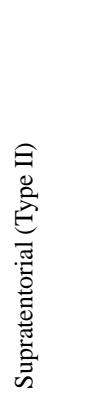 & 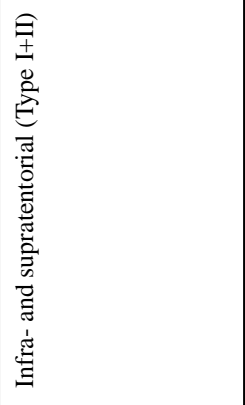 & 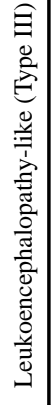 & 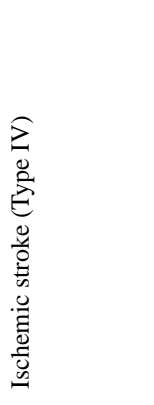 & 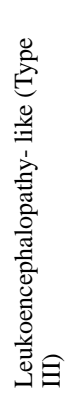 & 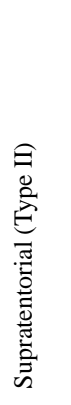 \\
\hline 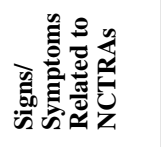 & 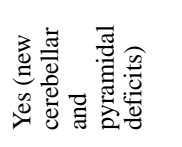 & 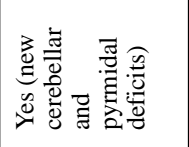 & $\stackrel{2}{z}$ & $\ddot{z}$ & 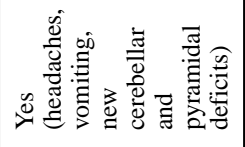 & ¿ & 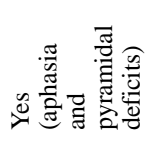 & $\stackrel{\circ}{z}$ & z \\
\hline 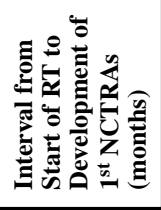 & 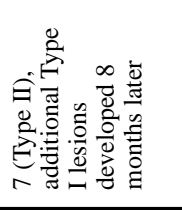 & 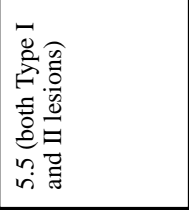 & 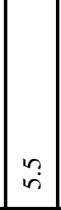 & 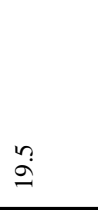 & 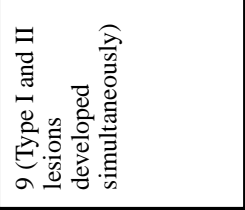 & 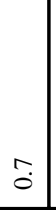 & $\stackrel{n}{\beth}$ & r & 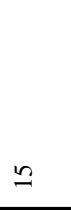 \\
\hline 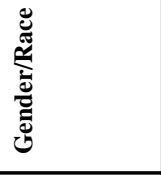 & $\coprod_{\Sigma}^{\circlearrowright}$ & $\underset{\mathbb{I}}{\mathbb{4}}$ & 宝 & $\sum_{i}^{\mathfrak{m}}$ & $\coprod_{\Sigma}$ & $\frac{\pi}{\Sigma}$ & $\stackrel{\bigcup}{\Psi}$ & $\stackrel{\bigcup}{\mathcal{I}}$ & 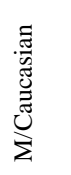 \\
\hline 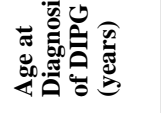 & $\vec{i}$ & $\stackrel{\bullet}{i}$ & $\ddot{m}$ & $\stackrel{\bullet}{\dot{m}}$ & $\stackrel{r}{r}$ & $\stackrel{ナ}{+}$ & $\stackrel{\circ}{+}$ & $\stackrel{r}{\dot{f}}$ & 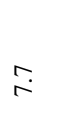 \\
\hline 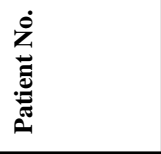 & - & $N$ & $n$ & + & in & 0 & r & $\infty$ & $a$ \\
\hline
\end{tabular}

